\title{
A method for construction of orbits in the gravitational field of a plane galactic disk
}

\author{
G. Malasidze and M. Todua
}

\author{
Georgian National Astrophysical Observatory \\ e-mail: maya@genao.org
}

Received 13 February 2007 / Accepted 26 September 2007

\begin{abstract}
We developed a new method for the construction of orbits in the field of gravitational potential of a plane galactic disk. On the basis of three isolating integrals of motion, the three invariants of motion were found as combinations of the elements of a Keplerian ellipse. The equation of so-called "blending ovals" representing the fourth-degree convex algebraic curve convenient for constructing the whole orbit was found. As an example, the values of the integrals of motion for a globular cluster Pal5 traveling along an almost plane meridional orbit were defined from the observational data, which were used to construct the blending contours of the cluster and its orbit. We also found a condition of periodicity for a certain class of orbits.
\end{abstract}

Key words. Galaxy: kinematics and dynamics - globular clusters: general - methods: analytical

\section{Introduction}

The first plane model of stationary, rotationally symmetric stellar systems with thriaxial ellipsoidal distribution of velocities and based on the gravitational potential

$\Phi(R, z)=G M\left(R^{2}+\left(|z|+z_{0}\right)^{2}\right)^{-1 / 2}$

was introduced by Kuzmin (1953a,b, 1956) and later by Toomre (1963), which we refer to as "the Kuzmin potential" in this paper. Here $G$ is the gravitational constant, $M$ the total mass of the model, $z_{0}$ a parameter with the dimension of length, and $R, z$, and $\theta$ are the cylindrical coordinates. For this model, alongside the energy integral $I_{1}$ and the integral of kinetic momentum $I_{2}$ relative to the symmetry axis, there is also the third isolating integral $I_{3}$, which is quadratic in the components of velocity. This model was investigated by Dejonghe \& de Zeeuw (1988b). It should be noted that the problem of the determination of orbits in the field of the potential (1) has solutions in elliptic functions (Malasidze 1982a).

From the expression of the potential (1), one can easily guess that the orbit should be presented formally as a set of segments of Keplerian orbits with foci at the points $\left(0 ; \pm z_{0}\right)$, where the total mass of the model is concentrated. For $z>0$ the center of gravity will be located at the point with the coordinates $\left(0 ;-z_{0}\right)$ and for $z<0-$ at $\left(0 ; z_{0}\right)$, i.e. mutual exchange of these centers of gravity can only occur when an orbit intersects the plane of symmetry $z=0$. These are the points where the pieces of ellipses merge so that smoothness of orbits is retained, and the acceleration changes discontinuously.

Many models developed by various researchers within the theory of the third isolating integral of motion, which is quadratic in velocities, were described in detail in V. Antonov's review papers (Antonov 1968, 1985) and I. King's monograph (King 2002). Orbits in Kuzmin potential were widely discussed in de Zeeuw's paper (1985). We would like to note the series of models built by Kuzmin \& Malasidze (1987), among which the models allowing the solution to the problem of determining three-dimensional orbits in special functions should be pointed out (Malasidze 1982b). The Kuzmin plane model with the gravitational potential (1) also belongs to this special case. Particle trajectories in the Kuzmin potential are also discussed in Tohline \& Voyages (2001).

The purpose of this paper is to develop a method for constructing orbits by "gluing" the pieces of different Keplerian ellipses for any point of the space of integrals of motion $\left(I_{1}, I_{2}, I_{3}\right)$. The interesting first results of the research in this direction can be found in the works by Kuzmin \& Malasidze (1990), Hunter (2002), and Kutuzov (2004).

\section{Selecting the invariants of motion}

The form of the Kuzmin gravitational potential (1) allows the existence of three isolating integrals of motion: the energy integral

$I_{1}=v_{R}^{2}+v_{\theta}^{2}+v_{z}^{2}-2 \Phi(R, z)$,

the integral of kinetic momentum relative to the symmetry axis of the model

$I_{2}=R v_{\theta}=R^{2} \mathrm{~d} \theta / \mathrm{d} t$,

and the third integral of motion, quadratic in components of velocity

$I_{3}=\left(R v_{z}-z v_{R}\right)^{2}+z^{2} v_{\theta}^{2}+z_{0}^{2}\left(v_{z}^{2}-2 \Phi^{\star}\right)$,

where $v_{R}=\mathrm{d} R / \mathrm{d} t, v_{\theta}=R \mathrm{~d} \theta / \mathrm{d} t$, and $v_{z}=\mathrm{d} z / \mathrm{d} t$ are the components of velocity, and the function $\Phi^{\star}$ satisfies the following conditions (Kuzmin 1956):

$$
\begin{aligned}
& z_{0}^{2} \partial \Phi^{\star} / \partial R=z^{2} \partial \Phi / \partial R-R z \partial \Phi / \partial z \\
& z_{0}^{2} \partial \Phi^{\star} / \partial z=\left(R^{2}+z_{0}^{2}\right) \partial \Phi / \partial z-R z \partial \Phi / \partial R
\end{aligned}
$$


One can easily see that the motion in the field of potential (1) is identical to the motion when all the mass of the system is concentrated consecutively at $\left(0, z_{0}\right)$ and $\left(0,-z_{0}\right)$, lieing on the symmetry axis of the model, so that the center of gravity would be at $\left(0,-z_{0}\right)$ for $z>0$, and for $z<0$ it would move to $\left(0, z_{0}\right)$.

Notice that in this case the orbit is presented as a set of pieces of different Keplerian ellipses, corresponding to the attracting foci in the points $\left(0, \pm z_{0}\right)$. At the intersection points of the orbit with the plane of symmetry $z=0$, the neighboring pieces will merge without changing the vector of corresponding velocity, while the vector of acceleration will change abruptly.

Even though Keplerian elements of various elliptic pieces in general will not be the same due to the existence of three isolating integrals $\left(I_{1}, I_{2}, I_{3}\right)$, there must be some combinations of these elements that will retain their values during the motion. We call such combinations of Keplerian elements $\left(c_{1}, c_{2}, c_{3}\right)$ the invariants of motion. Below we find the analytical expressions of these invariants and work out their relation to the integrals $\left(I_{1}, I_{2}, I_{3}\right)$.

In the Newtonian gravitational field of a point mass, for a double total energy of the moving object we have

$I_{1}=-G M / a$,

where $a$ is the semi-major axis of a Keplerian orbit. Thus, $a$ is one of the invariants of motion. For the first invariant we choose the expression

$c_{1}=z_{0}^{2} a^{-1}=-\left(z_{0}^{2} / G M\right) I_{1}$.

For the kinetic momentum relative to the axis of symmetry one can write:

$I_{2}^{2}=G M a\left(1-e^{2}\right) \cos ^{2} i=G M p \cos ^{2} i$,

where $e$ is the eccentricity of the Keplerian ellipse, $i$ the inclination angle of the plane of the ellipse to the plane of symmetry $z=0$, and $p=a\left(1-e^{2}\right)$. The second invariant of motion is expressed by

$c_{2}=p \cos ^{2} i=I_{2}^{2} / G M$.

To choose the third invariant of motion $c_{3}$ at $z=0$, the following relations can be used:

$1 / \sqrt{R^{2}+z_{0}^{2}}=(1+e \cos (u-\omega)) / p$,

$z_{0} / \sqrt{R^{2}+z_{0}^{2}}=\sin u \sin i$

where $u$ is a parameter, and $\omega$ the angle analogous to the argument of perihelion.

From these relations we get

$\left(z_{0} / p\right)(1+e \cos u \cos \omega+e \sin u \sin \omega)=\sin i \sin u$,

$\left(z_{0} / p\right)(1 / \sin u+e \cot u \cos \omega)=\sin i-\left(z_{0} / p\right) e \sin \omega$.

One can see that

$\left(z_{0} / p\right)\left(1 / \sin ^{2} u+2 e \cot u \cos \omega / \sin u+e^{2} \cot ^{2} u \cos ^{2} \omega\right)$

$=\left(p / z_{0}\right)\left(\sin i-\left(z_{0} / p\right) e \sin \omega\right)^{2}$.

It can be shown that at $z=0$

$v_{z} \sqrt{R^{2}+z_{0}^{2}}=z_{0} \sqrt{G M / p}(\cot u+e \cos \omega / \sin u)$.
Taking into account the value of the integral $I_{3}$ at $z=0$ (in the plane of symmetry), one can find that

$$
\begin{aligned}
& v_{z}^{2}\left(R^{2}+z_{0}^{2}\right) / G M z_{0}=\left(z_{0} / p\right)\left(\cot ^{2} u+2 e \cos \omega \cot u / \sin u\right. \\
& \left.\quad+e^{2} \cos ^{2} \omega / \sin ^{2} u\right)=c .
\end{aligned}
$$

Subtracting this expression from (13), one gets

$\left(z_{0} / p\right)\left(1-e^{2}-\left(p / z_{0}\right)^{2} \sin ^{2} i+2\left(p / z_{0}\right) e \sin i \sin \omega\right)=-c$,

from which the following expression can be chosen for the third invariant of motion:

$c_{3}=p-2 e z_{0} \sin i \sin \omega$.

For the third isolating integral we have

$I_{3}=G M\left(c_{3}-c_{1}-c_{2}\right)$.

\section{The method for describing the motion}

The three linearly independent invariants of motion, found in the previous section as combinations of Keplerian orbital elements, retain their constant values for all elliptical pieces on both sides of the plane of symmetry $z=0$. Let us write them in this form:

$c_{1}=\frac{z_{0}^{2}}{a}=\frac{z_{0}^{2}\left(1-e^{2}\right)}{p}$,

$c_{2}=p \cos ^{2} i$

$c_{3}=p-2 z_{0} e \sin i \sin \omega$

The parametric equations of each piece of the ellipse are

$s=\sqrt{R^{2}+\left(|z|+z_{0}\right)^{2}}=\frac{p}{1+e \cos (u-\omega)}$,

$\tan (\theta-\Omega)=\tan u \cos i$,

$|z|+z_{0}=s \sin u \sin i$

where $\Omega$ is the angle similar to the longitude of the ascending node, and the other notations are explained in the previous section.

For the elements $e, i$, and $\omega$, we have the following relations:

$z_{0}^{2} e^{2}=z_{0}^{2}-c_{1} p$

$p \sin ^{2} i=p-c_{2}$,

$\sin ^{2} \omega=\frac{p\left(p-c_{3}\right)^{2}}{4\left(p-c_{2}\right)\left(z_{0}^{2}-c_{1} p\right)}$.

The sign of $\sin \omega$ is defined by the expression (21). Using these relations, from (22), (23), (24) we obtain the equations

$$
\begin{aligned}
& \left(4 c_{1}\left(p-c_{2}\right)+\left(p-c_{3}\right)^{2}\right) s^{2}-4 z_{0}^{2}\left(p+c_{3}-2 c_{2}\right) s \\
& \quad+4 z_{0}^{2}\left(\left(c_{3}-c_{1}-c_{2}\right) p+z_{0}^{2}\right)=0 \\
& \sin (\theta-\Omega)=z_{0} \sqrt{c_{2} /\left(p-c_{2}\right)\left(s^{2}-z_{0}^{2}\right)},
\end{aligned}
$$

which completely solve the problem of linking separate ellipses and makes the construction of a whole orbit possible for every set of invariants $\left(c_{1}, c_{2}, c_{3}\right)$. Equation (28) represents a fourthdegree closed convex algebraic curve in the plane $(p, s)$, which 
we call "a blending oval". With the help of such ovals, it is easy to draw orbits of objects moving in the field of the potential (1). With the initial conditions of motion, for the starting value of $p$, we have

$G M p=s^{2} v_{\theta}^{2}+\left(\left(|z|+z_{0}\right) v_{R}-R v_{z}\right)^{2}$.

For every $p$ there is a corresponding pair of $\left(s_{1}, s_{2}\right)$, according to Eq. (28):

$s_{1,2}=\frac{2 z_{0}}{4 c_{1}\left(p-c_{2}\right)+\left(p-c_{3}\right)^{2}}\left(\left(p+c_{3}-2 c_{2}\right) z_{0}\right.$

$\left.\pm \sqrt{\left(c_{3}-c_{1}-c_{2}\right)\left(4\left(z_{0}^{2}-c_{1} p\right)\left(p-c_{2}\right)-p\left(p-c_{3}\right)^{2}\right)}\right)$,

and vice versa: for every $s$ there is a pair of $p$ values, according to the same equation. For periodic orbits, the number of pairs of $s$ and $p$ will be limited for a certain set of three invariants, while it will be unlimited in the case of non-periodic orbits.

Excluding the parameter $u$ from (22), (23), and (24), we obtain a new system of equations for corresponding segments of the orbit:

$(s-p) \sin i+e \cos \omega \sqrt{R^{2}-s^{2} \cos ^{2} i}$

$$
+e\left(|z|+z_{0}\right) \sin \omega=0,
$$

$\tan (\theta-\Omega)=\frac{\left(|z|+z_{0}\right) \cos i}{\sqrt{R^{2}-s^{2} \cos ^{2} i}}$

where $s=\sqrt{R^{2}+\left(|z|+z_{0}\right)^{2}}$. These segments will merge at the intersection of the orbits with the plane of symmetry of the model (galactic plane). At the same time, it must be noted that according to the classification of spacial stellar orbits (Antonov 1968), mainly tube orbits occur in the field of the potential (1) (see also de Zeeuw \& Hunter 1990).

\section{Periodic orbits}

Periodic orbits with equal periods in $R$ and $z$, or in elliptical coordinates $\xi_{1}$ and $\xi_{2}$, related as

$R=z_{0} \sqrt{\left(\xi_{1}^{2}-1\right)\left(1-\xi_{2}^{2}\right)}, \quad|z|=z_{0} \xi_{1}\left|\xi_{2}\right|$,

can be encountered when $\cos \omega=0$ (or $\sin \omega= \pm 1$ ). In this case the fourth invariant of motion appears:

$c_{4}=p+z_{0} e \csc i$.

This leads to the relations

$\left(p-c_{4}\right)\left(p-c_{3}\right)=2\left(z_{0}^{2}-c_{1} p\right)$,

$p-c_{3}=2\left(p-c_{4}\right) \sin ^{2} i$,

$p\left(p+c_{3}-2 c_{4}\right)=2 c_{2}\left(p-c_{3}\right)$

from which we obtain two equivalent equations:

$p^{2}-\left(c_{3}+c_{4}-2 c_{1}\right) p+c_{3} c_{4}-2 z_{0}^{2}=0$,

$p^{2}-\left(2 c_{2}+2 c_{4}-c_{3}\right) p+2 c_{2} c_{4}=0$.

Therefore, $c_{1}, c_{2}, c_{3}$ and $c_{4}$ satisfy the conditions

$2 c_{1}+2 c_{2}-2 c_{3}+c_{4}=0, \quad\left(c_{3}-2 c_{2}\right) c_{4}=2 z_{0}^{2}$.
Solving this system with respect to $c_{3}$ and $c_{4}$, we find

$c_{3}=\frac{1}{2}\left(c_{1}+2 c_{2}+\sqrt{\left(c_{1}-c_{2}\right)^{2}+4}\right)$

$c_{4}=c_{3}-c_{1}+\sqrt{\left(c_{1}-c_{2}\right)^{2}+4}$

For periodic orbits with equal periods, the invariants of motion $c_{1}, c_{2}$, and $c_{3}$ are bound by the condition

$\left(c_{3}-2 c_{2}\right)\left(c_{3}-c_{1}-c_{2}\right)=z_{0}^{2}$,

which directly follows from (41). This condition is equivalent to the existence of multiple roots of the quadratic Eq. (28) relative to $s$. In case $c_{2}=0$, i.e. at $\theta=$ const., the following equation can be found with respect to $p$ :

$\left(4 z_{0}^{2}-4 c_{1} p-\left(p-c_{3}\right)^{2}\right) p=0$

For the roots of this we have

$p=0, \quad p=c_{3}-2 c_{1} \pm 2 \sqrt{z_{0}^{2}+c_{1}^{2}-c_{1} c_{3}}$,

from which the two largest roots should be chosen. It is necessary that

$c_{1}\left(c_{3}-c_{1}\right) \leq z_{0}^{2} \quad$ or $\quad c_{3} \leq z_{0}^{2} / c_{1}+c_{1}$.

When $c_{1}>z_{0}$, at least one root of Eq. (45) will be negative, instead of which $p=0$ should be used. To ensure at least one non-negative root, the following must be true:

$c_{3} \leq 2 z_{0}$.

In the general case, when $c_{2} \neq 0$, we have the equation

$4\left(z_{0}^{2}-c_{1} p\right)\left(p-c_{2}\right)-p\left(p-c_{3}\right)^{2}=0$,

one of the roots of which will always be negative.

In the space of invariants $\left(c_{1}, c_{2}, c_{3}\right)$, the parametric equations of the envelope of the family of surfaces (49) can be written as

$p^{3}-2\left(c_{3}-2 c_{1}\right) p^{2}+\left(c_{3}^{2}-4\left(c_{1} c_{2}+z_{0}^{2}\right)\right) p+4 z_{0}^{2} c_{2}=0$

$3 p^{2}-4\left(c_{3}-2 c_{1}\right) p+c_{3}^{2}-4\left(c_{1} c_{2}+z_{0}^{2}\right)=0$.

The isolines of the invariants $c_{1}, c_{2}$, and $c_{3}$ will be straight lines, defined by the equations:

$p-c_{2}=p\left(c_{3}-c_{2}\right) / \sqrt{4 z_{0}^{2}+p^{2}}$,

$2\left(p c_{1}-z_{0}^{2}\right)=\left(1+\left(2 z_{0}^{2}+p^{2}\right) / p \sqrt{4 z_{0}^{2}+p^{2}}\right)\left(c_{3}-c_{1}\right)$.

The isolines $c_{2}$ can be easily drawn using these formulae. In the space of invariants, the isolines of the parameter $p$ are also straight lines (on the boundary surface). 


\section{An example of constructing the orbits}

Despite the extreme complexity and multicomponentness of the structure of our Galaxy, the gravitational potential of the regular galactic field, at least in a rough approximation can be expressed by (1), since the greatest part of the total observed mass is concentrated in the plane of symmetry - the disk. The potential (1) at $z=0$ becomes

$\Phi(R, 0)=\frac{G M}{z_{0}\left(1+R^{2} / z_{0}^{2}\right)^{1 / 2}}$,

according to which one finds for the rotational velocity $v$ and the Oort constant $A$ :

$v^{2}=-R \mathrm{~d} \Phi / \mathrm{d} R=G M R^{2} /\left(z_{0}^{3}\left(1+R^{2} / z_{0}^{2}\right)^{3 / 2}\right)$,

$A=-(1 / 2) R \mathrm{~d}(v / R) / \mathrm{d} R=(3 / 4) R v /\left(z_{0}^{2}\left(1+R^{2} / z_{0}^{2}\right)\right)$.

From these relations and observational data of the solar neighborhood,

$R_{0}=8.5 \mathrm{kpc}, \quad v_{0}=220 \mathrm{~km} \mathrm{~s}^{-1}$,

where $R_{0}$ is the distance of the Sun from the center of the Galaxy, $v_{0}$ the circular velocity of rotation of the solar centroid. The following parameters of the model can be assessed:

$z_{0}=R_{0} / \sqrt{2}=6 \mathrm{kpc}$,

$G M=(1.5)^{3 / 2} R_{0} v_{0}^{2}=755790 \mathrm{~km}^{2} \mathrm{~s}^{-2} \mathrm{kpc}$.

The boundaries of the region occupied by an orbit are determined by the values of the invariants $c_{1}, c_{2}$, and $c_{3}$ according to the equation

$\left(c_{1} \xi^{2}-2 z_{0}|\xi|+c_{3}-c_{1}-c_{2}\right)\left(\xi^{2}-1\right)+z_{0}^{2} c_{2} \xi^{2}=0$,

which, when $c_{2}=0$ ( $\theta=$ const. $)$, turns into a quadratic equation:

$c_{1} \xi^{2}-2 z_{0}|\xi|+c_{3}-c_{1}=0$.

Obviously, the roots are

$|\xi|=\left(z_{0} \pm \sqrt{z_{0}^{2}-c_{1}\left(c_{3}-c_{1}\right)}\right) / c_{1}$.

As an example of the application of our theory, we choose a spherical cluster Pal5, where all necessary data were taken from Dauphole et al. (1996). The values of all invariants of motion and the initial values of the orbital elements are presented in the table below:

\begin{tabular}{cc}
\hline \hline$v_{R}\left(\mathrm{~km} \mathrm{~s}^{-1}\right)$ & -124 \\
$v_{z}\left(\mathrm{~km} \mathrm{~s}^{-1}\right)$ & 64 \\
$c_{1} / z_{0}$ & 0.33 \\
$c_{2} / z_{0}$ & 0 \\
$c_{3} / z_{0}$ & 3.05 \\
$e$ & 0.33 \\
$i$ & 90 \\
$R / z_{0}$ & 1.3 \\
$z / z_{0}$ & 2.67 \\
$s / z_{0}$ & 3.9 \\
$\sin \omega$ & -0.53 \\
$\cos \omega$ & 0.85 \\
$p / z_{0}$ & 2.70 \\
\hline &
\end{tabular}

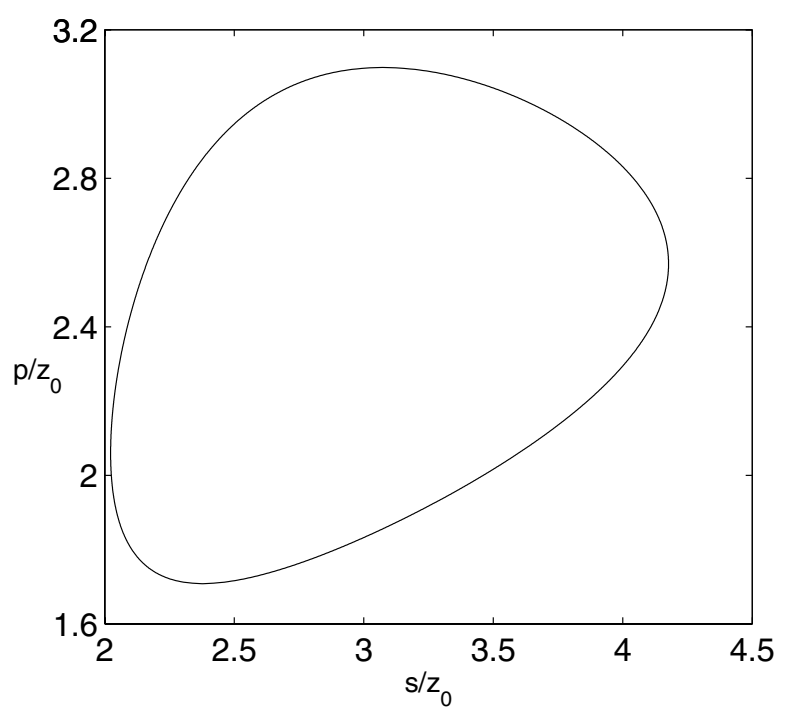

Fig. 1. The blending oval for the globular cluster Pal5 (formula (31)).

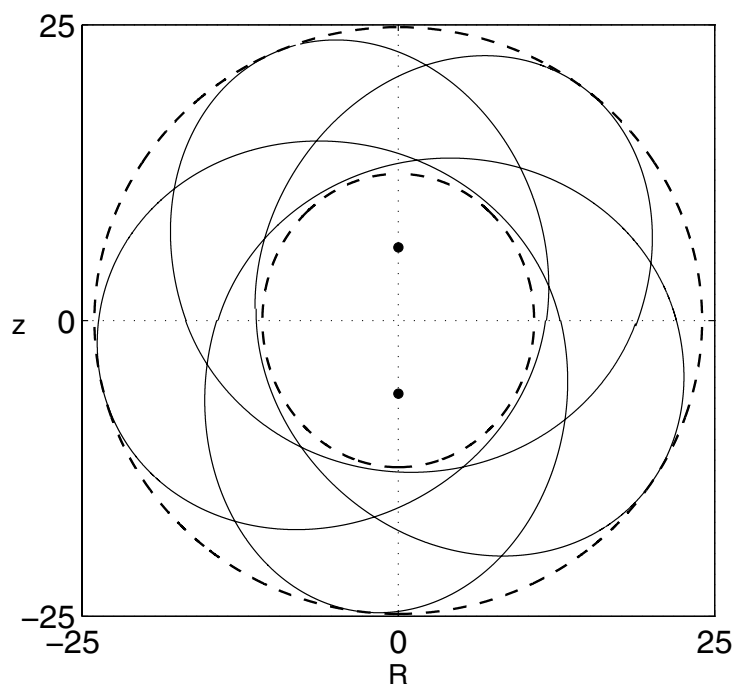

Fig. 2. Orbits of the globular cluster Pal5. The dashed ellipses bound the area of admissible motion ((62) and (63)). The bold dots mark the foci.

The blending oval calculated for Pal5 by (31) is presented in Fig. 1. The area of admissible motion for the cluster Pal5 is constrained by the ellipses:

$\frac{R^{2}}{15}+\frac{z^{2}}{16}=z_{0}^{2}$

$\frac{R^{2}}{3}+\frac{z^{2}}{4}=z_{0}^{2}$

These areas and some orbits, calculated using (32) and values from the blending oval, are presented in Fig. 2. Since the invariant $c_{2}=0$, the cluster orbits in a meridional plane around the Galactic center and is very similar to the orbit constructed by Dauphole \& Colin (1995), using completely different model.

\section{Conclusion}

Among the models with the third isolating integral of motion allowing a solution to the problem of triaxial orbits in special 
functions, the widely-tested Kuzmin model of the plane disk appeared to be extremely simple and a prospective for describing a motion. Finding the three independent invariants of motion as the simplest combinations of the elements of Keplerian orbits allowed us to develop a new graphical method for constructing orbits by contours (28) providing the merging of number of elliptical segments.

For a special class of orbits, the condition of periodicity was found (44), which in a general case should have a more complicated form. The isolines of some of the most important parameters of motion are also revealed.

Using the observational data, the main parameters of the model of a plane disk were estimated. A so-called blending oval was drawn for the spherical cluster Pal5, which travels along an approximate plane meridional orbits. These orbits were drawn using the blending oval. It was found that the cluster can approach the center of the Galaxy no closer than $11 \mathrm{kpc}$. One could say with confidence that these results fully agree with the study by Dauphole et al. (1996) of the motion of the cluster Pal5 obtained on the basis of the three-component model of the Galaxy.

\section{References}

Antonov, V. A. 1968, Itogi Nauki i Tekhniki. Seria Astron., 61, 93 Antonov, V. A. 1985, Itogi Nauki i Tekhniki. Seria Astron., 26, 4 Dauphole, B., \& Colin, J. 1995, A\&A, 300, 117

Dauphole, B., Geffert, M., Colin, J., et al. 1996, A\&A, 313, 119

Dejonghe, H., \& de Zeeuw, P. T. 1988a, ApJ, 329, 720

Dejonghe, H., \& de Zeeuw, P. T. 1988b, ApJ, 333, 90

de Zeeuw, P. T. 1985, MNRAS, 216, 273

de Zeeuw, P. T., \& Hunter, C. 1990, ApJ, 356, 365

Hunter C. 2002, Disk-crossing orbits, private communication, a talk in Athens, to be published by Springer in their Lecture Notes in Physics series, 1.7

King, A. R. 2002, Introduction to Classical Stellar Dynamics

Kutuzov, S. A. 2004, ASP Conf. Proc., 316, 37

Kuzmin, G. G. 1953a, Izvestia AN Estonii, 2, 368

Kuzmin, G. G. 1953b, Publ. Tartu Astron. Obs., 32, 332

Kuzmin, G. G. 1956, Astron. Zhurnal, 33, 27

Kuzmin, G. G., \& Malasidze, G. A. 1987, Publ. Tartu Astron. Obs., 52, 48

Kuzmin, G. G., \& Malasidze, G. A. 1990, Voprosy Nebesnoy Mekhaniki i Zvezdnoy Dinamiki. Alma Ata, Nauka 107

Malasidze, G. A. 1982a, Bull. Abastumani Astrophys. Obs., 55, 197

Malasidze, G. A. 1982b, Bull. Abastumani Astrophys. Obs., 55, 203

Tohline, J. E., \& Voyages, K. 2001, ApJ, 555, 524

Toomre, A. 1963, ApJ, 138, 385 